\title{
PLANILHA EXCEL/VBA PARA ESTIMATIVAS DE COEFICIENTES DE ATIVIDADE DE MISTURAS USANDO CONTRIBUIÇÕES DE GRUPOS FUNCIONAIS
}

\author{
T. Q. Oliveira, T. D. Romão, L. G. Oliveira, A. G. Cabral, S. P. Nascimento, J. A. Cavalcante, \\ N. A. Costa
}

Universidade Federal da Paraíba, Departamento de Engenharia Química

E-mail para contato: nagelalves@hotmail.com

\begin{abstract}
RESUMO - O trabalho tem por finalidade apresentar uma planilha eletrônica com programação em objeto-modelo VBA para estimativas de coeficientes de atividade e energia livre de Gibbs adimensional de misturas multicomponentes usando o modelo de contribuições de grupos funcionais UNIFAC. O aplicativo foi codificado na forma modular: a) banco de dados contendo os parâmetros de interação binária grupo-grupo, parâmetros de área e de volume molecular de 108 grupos funcionais; b) criação de caixas de diálogos para interação do usuário com o aplicativo; c) codificações de subprogramas VBA para as estimativas dos coeficientes de atividade e energia livre de Gibbs em excesso adimensional e d) validação dos resultados fornecidos pelos subprogramas codificados. O resultado final é um aplicativo, para fins educacionais e/ou comerciais, capaz de fornecer estimativas dos coeficientes de atividades e energia livre de Gibbs em excesso com rapidez e confiabilidade.
\end{abstract}

\section{INTRODUÇÃO}

O modelo UNIFAC (Uniquac Functional Group Activity Coefficients) foi desenvolvido por Fredenslund et al. (1975) com a finalidade de prever coeficientes de atividade de componentes abaixo do ponto crítico, temperatura entre 300 e $425 \mathrm{~K}$, pressão moderada até 5 atm, líquidos miscíveis, misturas não eletrolíticas e não poliméricas. Este método considera que o coeficiente de atividade dos componentes em uma mistura líquida consiste de uma contribuição combinatorial (considera a não idealidade da fase líquida devido às diferenças no tamanho e forma dos grupos funcionais) e uma contribuição residual (considera a não idealidade da fase líquida devido às interações intermoleculares entre grupos funcionais). $\mathrm{O}$ modelo UNIFAC combina dois modelos: UNIQUAC (Universal Quasi Chemical) fornece à parte combinatorial e ASOG (Analitycal Solution of Groups) fornece à parte residual.

$$
\ln \gamma_{i}=\ln \gamma_{i}^{C}+\ln \gamma_{i}^{R}
$$

onde $\ln \gamma_{i}^{C}$ e $\ln \gamma_{i}^{R}$ são, respectivamente, os logaritmos das contribuições combinatorial e residual.

A parte combinatorial ou configuracional é determinada somente pela composição, pelo tamanho e forma das moléculas, requerendo somente dados dos componentes puros. 
$\ln \gamma_{i}^{C}=\ln \frac{\phi_{i}}{x_{i}}+\frac{Z}{2} q_{i} \ln \frac{\theta_{i}}{\phi_{i}}+L_{i}-\frac{\phi_{i}}{x_{i}} \sum_{j} x_{j} L_{j}$

Os parâmetros envolvidos na Equação (2) são descritos a seguir:

- O parâmetro de volume do grupo funcional $k, R_{k}: R_{\mathrm{k}}=\frac{\text { volume de van der Waals }}{15,17}$, onde o fator 15,17 é determinado pelo volume de $\mathrm{CH}_{2}$ no polietileno.

- $\mathrm{O}$ parâmetro de área superficial do grupo funcional $k, Q_{k}$ : $Q_{\mathrm{k}}=\frac{\text { área de van der Waals }}{2,5 \times 10^{9}}$, onde o fator $2,5 \times 10^{9}$ é determinado área superficial externa de $\mathrm{CH}_{2}$ no polietileno.

- Parâmetro de volume da molécula $i: r_{i}=\sum_{k} v_{k}^{i} R_{k}$, onde $v_{k}^{i}$ é o número de grupos do tipo $k$ na molécula $i$.

- Parâmetro de área superficial da molécula $i: q_{i}=\sum_{k} v_{k}^{i} Q_{k}$, onde $v_{k}^{i}$ é o número de grupos do tipo $k$ na molécula $i$.

- Fração de volume da espécie $i: \phi_{i}=\frac{r_{i} x_{i}}{\sum_{j} r_{j} x_{j}}$, onde $x_{i}$ é a fração molar da espécie $i$.

- Fração de área da espécie $i: \theta_{i}=\frac{q_{i} x_{i}}{\sum_{j} q_{j} x_{j}}$, onde $x_{i}$ é a fração molar da espécie $i$.

- Número de coordenação Z: representa o número de moléculas vizinhas a considerada, os autores usaram $Z=10$.

- Parâmetro auxiliar $L_{\mathrm{i}}: L_{i}=\frac{Z}{2}\left(r_{i}-q_{i}\right)-\left(r_{i}-1\right)$

A parte residual é determinada por:

$$
\ln \gamma_{i}^{R}=\sum v_{k}^{(i)}\left(\ln \Gamma_{k}-\ln \Gamma_{k}^{(i)}\right)
$$

onde $\Gamma_{k}$ é o coeficiente de atividade residual do grupo $k$ na solução e $\Gamma_{k}^{(i)}$ é o coeficiente de atividade residual do grupo $k$ em uma solução de referência consistindo somente de moléculas do componente $i$.

Os parâmetros $\Gamma_{k}$ são obtidos através da seguinte expressão:

$$
\ln \Gamma_{k}=Q_{k}\left[1-\ln \sum_{m} \theta_{m} \Psi_{m k}-\sum_{m} \frac{\theta_{m} \Psi_{k m}}{\sum_{n} \theta_{n} \Psi_{n m}}\right]
$$

A Equação (4) também é empregada para a predição do parâmetro $\ln \Gamma_{\mathrm{k}}^{(\mathrm{i})}$. 
Os parâmetros envolvidos na Equação (4) são descritos a seguir:

- Parâmetro $X_{m}: X_{m}=\frac{\sum_{i} x_{i} v_{m}^{i}}{\sum_{j} x_{j} \sum_{k} v_{k}^{j}}$ é a fração molar do grupo $m$ na solução líquida

- Fração molar de área do grupo $m: \theta_{\mathrm{m}}=\frac{Q_{\mathrm{m}} X_{\mathrm{m}}}{\sum_{\mathrm{n}} Q_{\mathrm{n}} X_{\mathrm{n}}}$

- Fração de área do grupo $m: \theta_{\mathrm{m}}=\frac{Q_{\mathrm{m}} X_{\mathrm{m}}}{\sum_{\mathrm{n}} Q_{\mathrm{n}} X_{\mathrm{n}}}$

Os parâmetros de interação binária entre grupos funcionais $m$ e $n$ são dados por:

$$
\Psi_{m n}=\exp \left(-\frac{U_{m n}-U_{n n}}{R T}\right)=\exp \left(-\frac{a_{m n}}{T}\right)
$$

onde $U_{m n}$ é uma medida da energia de interação entre os grupos funcionais $m$ e $n$. Os parâmetros de interação entre grupos funcionais $a_{m n}$ são obtidos de dados experimentais de equilíbrio entre fases.

\subsection{Coeficiente de Atividade à Diluição Infinita}

O coeficiente de atividade a diluição infinita representa a não idealidade máxima de um componente em solução, pois se refere ao limite de diluição em que uma molécula de um componente se encontra rodeada por várias moléculas do outro componente (Azevedo, 2011). Matematicamente: $\gamma_{i}^{\infty}=\lim _{x_{i} \rightarrow 0} \gamma_{i}$ ou $\ln \gamma_{i}^{\infty}=\lim _{x_{i} \rightarrow 0}\left(\ln \gamma_{i}\right)$. Aplicando esta condição na Equação (1), obtém-se:

$$
\ln \gamma_{i}^{\infty}=\lim _{x_{i} \rightarrow 0}\left(\ln \gamma_{i}\right)=\lim _{x_{i} \rightarrow 0}\left(\ln \gamma_{i}^{C}\right)+\lim _{x_{i} \rightarrow 0}\left(\ln \gamma_{i}^{R}\right)
$$

No limite quando a fração molar da espécie i tende a zero, a parte combinatorial é modificada para fornecer:

$$
\ln \gamma_{i}^{C}=\ln \left(\frac{r_{i}}{\sum_{j} r_{j} x_{j}}\right)+\frac{z}{2} q_{i} \ln \left[\left(\frac{q_{i}}{r_{i}}\right)\left(\frac{\sum_{j} r_{j} x_{j}}{\sum_{j} q_{j} x_{j}}\right)\right]+L_{i}-\left(\frac{r_{i}}{\sum_{j} r_{j} x_{j}}\right) \sum_{j} x_{j} L_{j}
$$

onde $\frac{\phi_{i}}{x_{i}}=\frac{q_{i}}{\sum_{j} q_{j} x_{j}}$ e $\frac{\phi_{i}}{\theta_{i}}=\left(\frac{\sum_{j} r_{j} x_{j}}{\sum_{j} q_{j} x_{j}}\right)\left(\frac{q_{i} x_{i}}{r_{i} x_{i}}\right)=\frac{q_{i} \sum_{j} r_{j} x_{j}}{r_{i} \sum_{j} q_{j} x_{j}}$. A expressão para a parte residual é idêntica à apresentada na Equação (3). 


\section{METODOLOGIA}

A metodologia adotada para o desenvolvimento dos subprogramas JAVA é esquematizada na Figura 1.

Figura 1 - Metodologia para o desenvolvimento do aplicativo

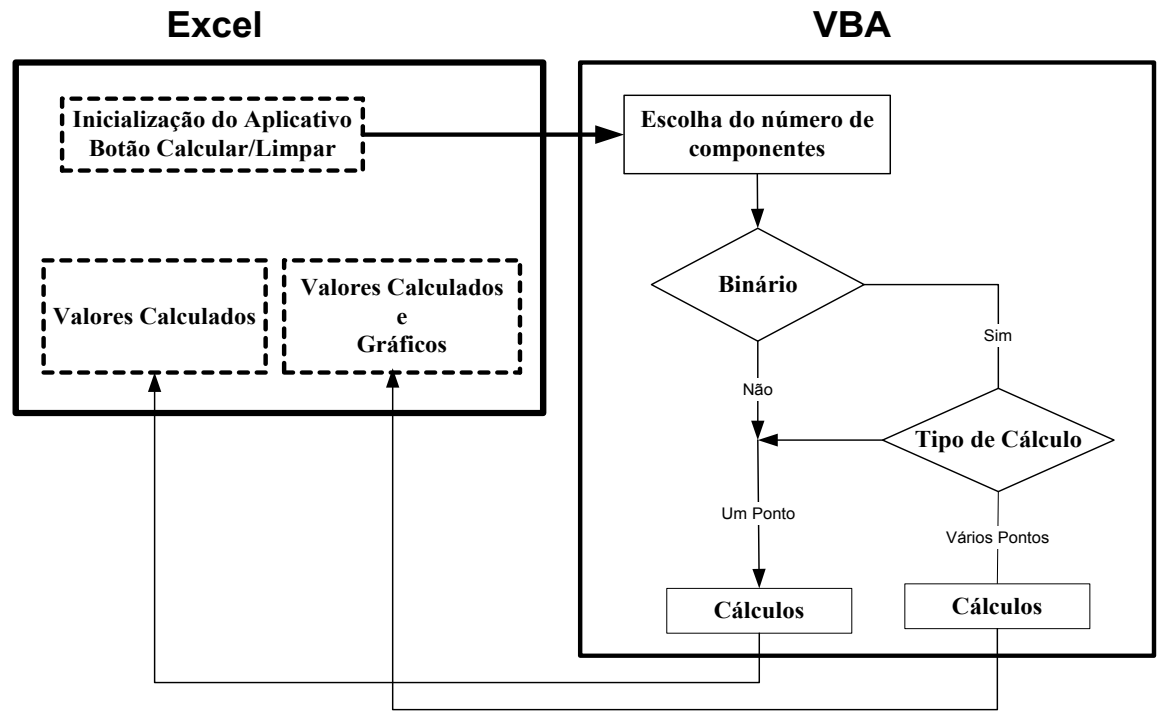

\subsection{Esquema do desenvolvimento do aplicativo}

As etapas do desenvolvimento do aplicativo Excel/VBA são descritas a seguir:

Banco de dados: $\mathrm{O}$ banco de dados contém os parâmetros de volume e de área de 108 grupos funcionais secundários e uma matriz 50 por $50 \mathrm{com}$ os parâmetros binários entre os grupos funcionais principais do modelo UNIFAC. Os valores utilizados nos bancos de dados são reportados por Fredenslund et al. (1977) e Fredenslund e Sorensen (1994).

Planilha: A Figura 2 mostra a planilha usada para iniciar as macros codificadas no VBA.

Figura 2 - Planilha utilizada para a configuração (cálculos) e saída de dados

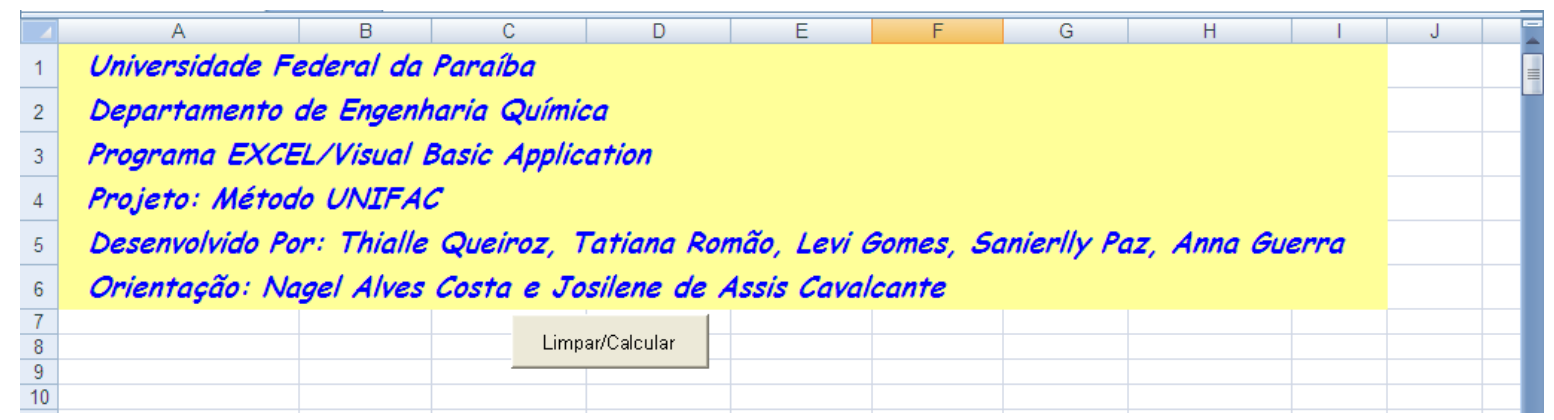

Escolha da quantidade de espécies químicas: A Figura 3 mostra a caixa de diálogo que permite ao usuário escolher o tipo de cálculo.

Escolha do tipo de cálculo: A Figura 4 mostra a caixa de diálogo que permite ao usuário escolher o tipo de cálculo. 
XI Congresso Brasileiro de Engenharia

Química em Iniciação Científica

Unicamp - Campinas - SP

19 a 22 de julho de 2015

Figura 3 - Escolha de Espécies

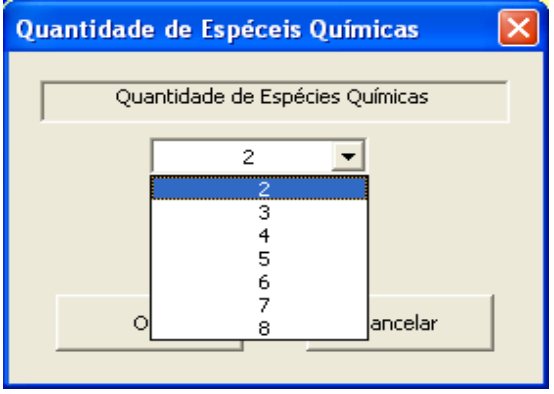

Figura 4 - Escolha do Tipo de Cálculo

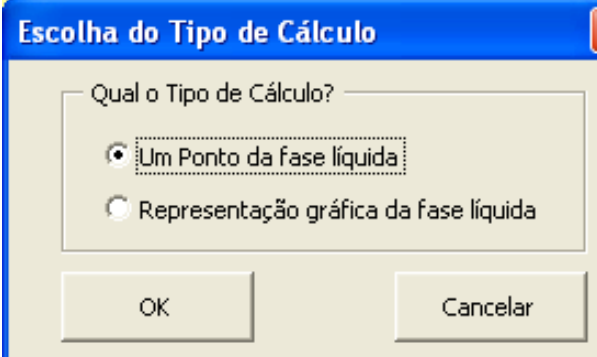

Configuração da espécie: A configuração é realizada de acordo com a Figura 5.

Quantidade de grupos funcionais da espécie: A quantidade de grupos funcionais da espécie é realizada de acordo com a Figura 6.

Figura 5 - Configuração da espécie

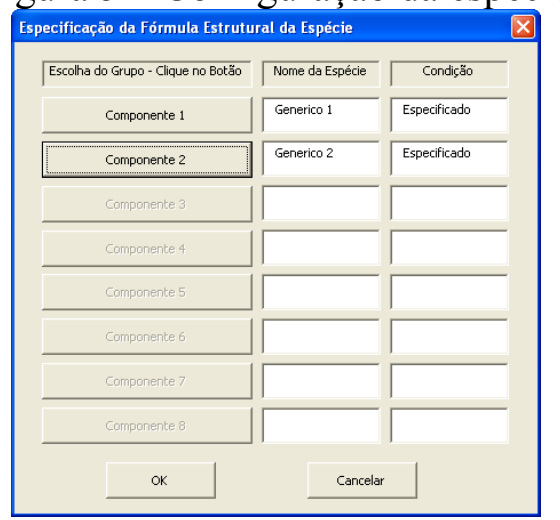

Figura 6-Quantidade de grupos funcionais

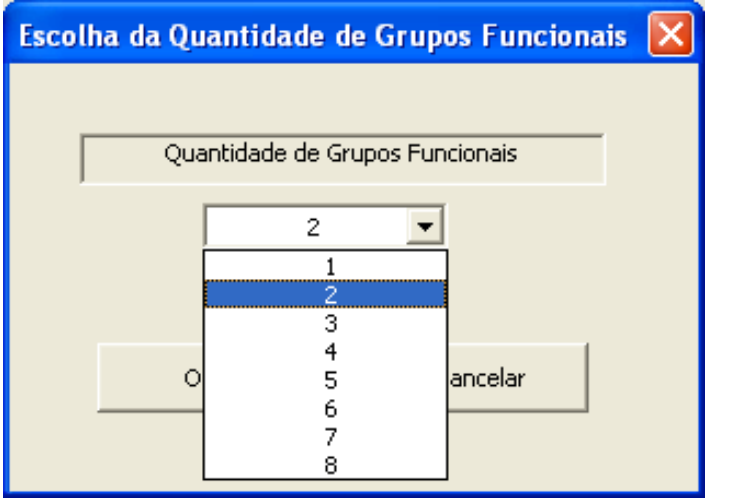

Escolha dos grupos funcionais: A escolha do grupo funcional é realizada de acordo com a Figura 7.

Quantidade de grupos funcionais da espécie: A quantidade de grupos funcionais da espécie é realizada de acordo com a Figura 8.

Especificações: As especificações são realizadas de acordo com a Figura 9.

Figura 7 - Escolha grupos

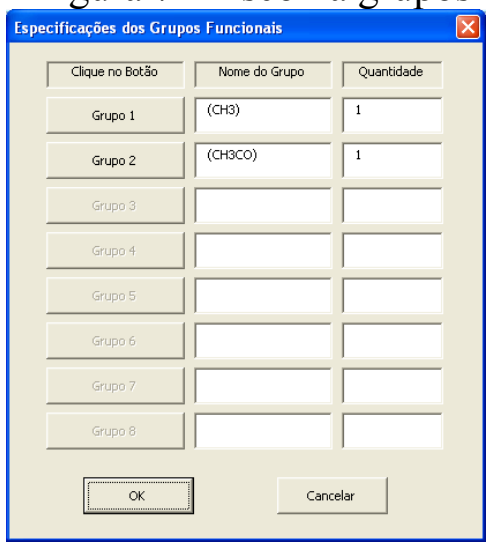

Figura 8 - Seleção grupos Escolha dos Grupos Funcionais

\begin{tabular}{|ll|}
\hline$(\mathrm{CH} 3)$ & \\
\hline$(\mathrm{CH} 3)$ & \\
\hline$(\mathrm{CH} 2)$ & \\
$(\mathrm{CH})$ \\
$(\mathrm{CCC})$ \\
$(\mathrm{CH} 2=\mathrm{CH})$ \\
$(\mathrm{CH}=\mathrm{CH})$ \\
$(\mathrm{CH} 2=\mathrm{C})$ \\
$(\mathrm{CH}=\mathrm{C})$ \\
$(\mathrm{C=C})$ \\
$(\mathrm{ACH})$ \\
$(\mathrm{AC})$ \\
$(\mathrm{ACCH} 3)$ \\
$(\mathrm{ACCH} 2)$ \\
$(\mathrm{ACCH})$ \\
$(\mathrm{OH})$ \\
\hline
\end{tabular}

Figura 9 - Especificações

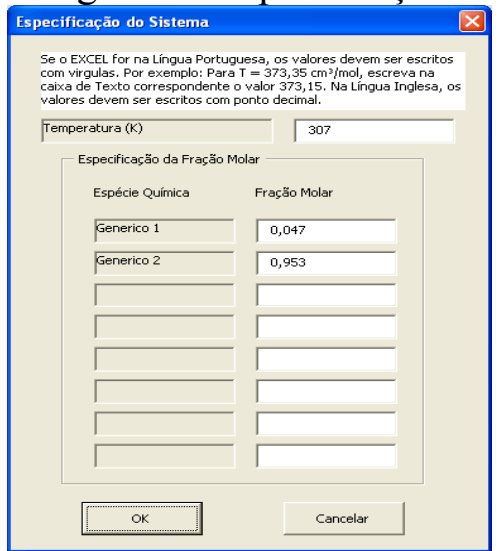




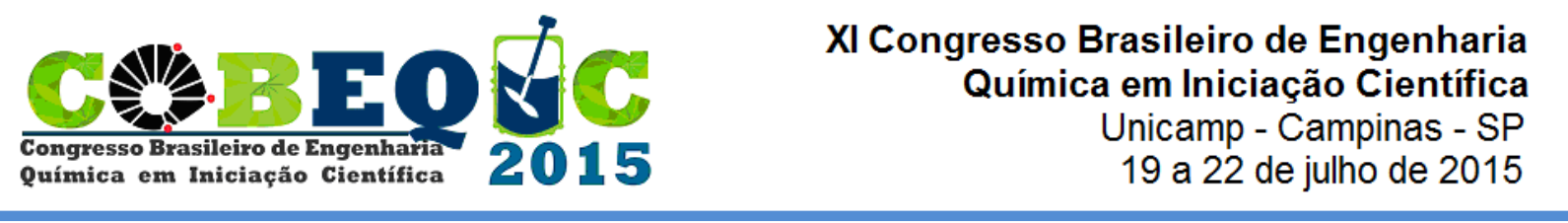

\section{VALIDAÇÃO DO APLICATIVO}

Vários sistemas reportados foram utilizados para a validação do aplicativo. Alguns resultados são apresentados na Tabela 1. A simbologia (exp), (F), (TE) e (calc) são, respectivamente, os coeficientes de atividade experimental, calculado por Fredenslund et. al. (1977), valores negrito reportados por Thomas e Eckert (1984) e calculado pelo aplicativo.

Tabela 1 - Coeficiente de atividade

\begin{tabular}{lccccc}
\hline Sistema & $\mathrm{T}(\mathrm{K})$ & $x_{1}$ & $\gamma_{1}(\mathrm{exp})$ & $\gamma_{1}(\mathrm{~F})$ & $\gamma_{1}$ (calc.) \\
\hline 1-Hexeno-Hexano & 333,2 & 0,121 & 1,022 & 1,024 & 1,027 \\
Hexano-1-Hexeno & 333,2 & 0,123 & 1,026 & 1,031 & 1,029 \\
Benzeno-Hexano & 298,2 & 0,100 & 1,550 & 1,478 & 1,478 \\
Hexano-Benzeno & 298,2 & 0,100 & 1,834 & 1,630 & 1,629 \\
Heptano-Tolueno & 383,7 & 0,000 & 1,411 & 1,541 & 1,499 \\
Tolueno-Heptano & 371,5 & 0,006 & 1,677 & 1,441 & 1,427 \\
Ciclohexano-CS $_{2}$ & $\mathbf{2 9 8 , 2}$ & $\mathbf{0 , 0 0 0}$ & $\mathbf{1 , 5 9}$ & $\mathbf{1 , 5 6}$ & 1,56 \\
Acetonitrila-CCl $_{4}$ & $\mathbf{2 9 3 , 2}$ & $\mathbf{0 , 0 0 0}$ & $\mathbf{1 3 , 4 0}$ & $\mathbf{1 1 , 1 7}$ & 11,17 \\
Acetona-CCl $_{4}$ & $\mathbf{3 0 3 , 9}$ & $\mathbf{0 , 0 0 0}$ & $\mathbf{3 , 0 3}$ & $\mathbf{2 , 9 3}$ & 2,93 \\
Hexano-Clorofórmio $_{\text {Benzeno-Acetonitrila }}$ & $\mathbf{3 1 9 , 8}$ & $\mathbf{0 9 0 0 0}$ & $\mathbf{1 , 7 9}$ & $\mathbf{1 , 8 5}$ & 1,85 \\
Heptano-Etanol $^{\mathbf{2 9 , 2}}$ & $\mathbf{0 , 0 0 0}$ & $\mathbf{3 , 1 9}$ & $\mathbf{3 , 1 3}$ & 3,13 \\
\hline
\end{tabular}

\section{CONCLUSÃO}

O programa foi exaustivamente testado para a verificação dos valores calculados. Os resultados indicaram que a planilha eletrônica com programação VBA fornece estimativas com confiabilidade e rapidez para o coeficiente de atividade através do método de contribuição de grupos funcionais UNIFAC. As pequenas diferenças verificadas são devido às diferentes precisões numéricas entre os aplicativos e os valores da literatura.

\section{REFERÊNCIAS}

AZEVEDO, E. G. Termodinâmica Aplicada. $3^{\text {a }}$ Edição, Escolar Editora, 2011.

FREDENSLUND, A. GMEHLING, J. RASMUSSEN, P. Vapor-Liquid equilibria using UNIFAC - a group-contribution method. Elsevier, 1977.

FREDENSLUND, A., JONES, R. L. PRAUSNITZ, J. M. Group-Contribution Estimation of Activity Coefficients in Nonideal Liquid Mixtures. AlChE Journal. v. 21, n. 6, p. 1086. 1975.

FREDENSLUND, A. SORENSEN, J. M. Group Contribution Estimation Methods. In: Models for Thermodynamic and Phase Equilibria Calculations. Edited SANDLER, I. S. Marcel Dekker, Inc. 1994.

THOMAS, E. R; ECKERT, C. A. Prediction Activity Coefficients by a Modified Separation of Cohesive Energy Density Model and UNIFAC. Ind. Eng. Chem. Process Des. Dev. 1984, 23, $198-204$. 